\title{
Endomysial IgA Antibody Measurement
}

National Cancer Institute

\section{Source}

National Cancer Institute. Endomysial IgA Antibody Measurement. NCI Thesaurus. Code C147334.

The determination of the amount of endomysial IgA antibody present in a sample. 